Review Article

\title{
Opioids in the World of Oral and Maxillofacial Surgery- A Review
}

\section{Muraleemohan $C^{1}$, Pratiksha Shetty ${ }^{2}$, Satadru Roy ${ }^{3}$, B. Rajendra Prasad ${ }^{4}$}

${ }^{1}$ Professor, M angalore, ${ }^{2}$ Assistant Professor, ${ }^{3}$ Post Graduate Student, Department of Oral \& M axillofacial Surgery, ${ }^{4}$ Director, P.G. Studies, A.B. Shetty Memorial Institute of Dental Sciences, Nitte (deemed to be University), Mangalore - 575018, Karnataka, India.

Corresponding Author: Satadru Roy, Post Graduate Student, Department of Oral \& M axillofacial Surgery, A.B. Shetty M emorial Institute of Dental Sciences, Nitte University, M angalore - 575018, Karnataka, India. M obile : +91 8584868501 , E-mail : drsataroy@ gmail.com

\section{Received : $\quad: 23.01 .2018$ \\ Review Completed : 26.03.2018 \\ Abstract:}

Accepted $\quad: 28.06 .2018$

Keywords : Opioids, Oral, Maxillofacial, Orthognathic.

\begin{tabular}{|c|}
\hline Access this article online \\
\hline Quick Response Code \\
\hline \\
\\
\end{tabular}

\begin{abstract}
Pain control is one of the most difficult challenges in medicine and a key facet of disease management. The isolation of Morphine by Friedrich Sertürner in 1804 added an essential pharmacological tool in the treatment of pain and spawned the discovery of a new class of drugs known collectively as Opioid analgesics. To date, Morphine and other opioids remain essential analgesics for alleviating pain. However, their use is plagued by major side effects, such as analgesic tolerance (diminished pain-relieving effects), hyperalgesia (increased pain sensitivity), and drug dependence. Despite the potential side effects, opioids remain the pharmacological cornerstone of modern pain therapy. Opioids are particularly effective for treating acute moderate-to-severe pain after surgery or trauma. Just like other disciplines of Medicine, pain management is an integral part in Oral and Maxillofacial Surgery. The wide domain of diseases and the procedures involved in treating them, require profound analgesia for sound patient management. In order to achieve that, Opioid analgesics play a vital role for providing long term pain free period, thus enjoying a widespread use in this field. Critical to the employment of Opioid therapy are proper patient evaluation and use of comprehensive management strategies. If certain criteria are followed, administration of oral opioids may be a successful means of decreasing the patient's debilitating chronic pain to tolerable levels, enabling an improvement in the quality of life and return to function. This article is an attempt to review the commonly used opioids and their extensive application in routine maxillofacial surgery practice.
\end{abstract}

\section{Introduction}

Opioids have been used as analgesic medications for centuries and are the cornerstone for the management of moderate to severe acute pain. ${ }^{[1],[2], ~[3]} \mathrm{M}$ any opioids are available for medical use, but the pharmacological action of all of them is very similar. They only differ with respect to their relative efficacy, pharmacokinetics and other drugspecific actions (eg. Codeine being used as an anti-tussive agent). Their activity is reversed by an opioid antagonist such as naloxone ${ }^{[4]}$, a highly specific, high-affinity opioid antagonist which can be safely administered by laypersons via intramuscular or intranasal routes, with virtually no side effects and no effect in the absence of opioids.

Traditionally, opioids are classified as weak, intermediate or strong according to their analgesic activity and propensity to addiction. Codeine is described as a weak opioid although at high dose can cause respiratory depression so the classification is not about safety ${ }^{[4]}$ It is to be noted that Opioids produce excellent analgesia without loss of sensations of touch, proprioception and consciousness. Patients who are given opioids alone will still retain awareness of the procedure and their memory will remain clear. ${ }^{[5]}$

Just like other M edical disciplines, M anagement of acute pain is essential to adequately treat oral and maxillofacial surgery patients. As a medication to control acute pain caused by oral and maxillofacial surgical procedures, opioids are prescribed in combination with acetaminophen or NSAIDs (including aspirin) and administered orally. In a hospital setting, parenterally administered opioids are more common, with novel methods of delivery; patient-controlled analgesia has been 
used frequently and has proved to be ef? cacious.

Patient Controlled Analgesia (PCA)refers to the methods of pain relief that use electronic or disposable devices and allow patients to self-administer analgesic drugs. While PCA may be used for a variety of analgesic groups, they have been most commonly used for morphine. Opioid consumption may be higher when comparing PCA with conventional administration but the incidence of side effects is the same. ${ }^{[4]}$

\section{Morphine}

M orphine is still the most commonly used opioid for acute pain. It derives its name from Morpheus, the Greek god of dreams. Morphine remains the gold standard with which other opioids are compared.

The absorption of morphine by mouth is variable; therefore, it is commonly given by intravenous or intramuscular injection. M ost morphine like drugs undergo considerable first pass metabolism and are therefore markedly less potent when taken orally than when injected. The half-life of morphine is $2-3$ ? hours. Hepatic metabolism is the main mode of inactivation, while metabolites are excreted in urine. ${ }^{[4]}$

\section{Codeine}

Codeine (3-methylmorphine) is useful in controlling mild to moderate pain. The drug is well absorbed orally when compared with morphine but has a low affinity for opioid receptors. Approximately, $10 \%$ of the drug is demethylated in the liver to morphine, which is responsible for the analgesic effects. The analgesic effect does not increase appreciably at higher doses. Codeine is also used as an antitussive and less commonly as an antidiarrhoeal agent.

\section{Tramadol}

Tramadol hydrochloride is a centrally acting synthetic opioid analgesic used to treat moderate to severe pain. Indian markets are flushed with over the counter, fixed dose combinations of NSAIDS, alongwith weaker opioids (like tramadol).It has selectivity for the $\mu$-receptor and a weak inhibitor of the reuptake of noradrenaline and serotonin. This resembles the action of tricyclic antidepressants and tramadol potentiates descending inhibitory pathways. This action has proven efficacy in the management of chronic pain.Tramadol does not have clinically significant respiratory depressant effect, and although generally well tolerated, it may cause nausea and vomiting, dizziness and drowsiness. In many countries, tramadol is not a controlled drug because it has a low potential for abuse and addiction. Tramadol has been used for pain after oral surgery. Tramadol undergoes hepatic metabolism, with metabolites being excreted by the kidneys. It has a half-life of 7 ? hours. ${ }^{[4]}$ It is not recommended for children under 12 years old

OPIOIDS THERAPEUTIC DOSAGE CHART

\begin{tabular}{|c|c|c|c|}
\hline $\begin{array}{l}\text { OPIOID } \\
\text { Route of administration }\end{array}$ & MORPHINE & CODEINE & TRAMADOL \\
\hline ORAL & $\begin{array}{l}0.3 \mathrm{mg} / \mathrm{kg} \text { every } 3-4 \text { hourly } \\
\text { Not to exceed } 1.6 \text { grams per day }\end{array}$ & $\begin{array}{l}30-60 \mathrm{mg} 4^{\text {th }} \text { hourly } \\
\text { Maximum dose- } 360 \mathrm{mg} \\
\text { in } 24 \text { hours }\end{array}$ & $\begin{array}{l}50-100 \mathrm{mg} 4^{\text {th }} \text { hourly } \\
\text { Maximum dose- } 400 \mathrm{mg} \text { in } \\
24 \text { hours }\end{array}$ \\
\hline IV & $\begin{array}{l}\text { 4-15 mg every } 3-4 \text { hours } \\
\text { Continuous dose- } 0.8-10 \mathrm{mg} / \text { hour } \\
\text { Maintainance dose- } \\
0.8-80 \mathrm{mg} / \text { hour }\end{array}$ & Not used via this route normally & $\begin{array}{l}50-100 \text { mg every } 4-6 \text { hours } \\
\text { Maximum dose- } 400 \text { mg } \\
\text { n } 24 \text { hours }\end{array}$ \\
\hline IM/SC & $\begin{array}{l}\text { Continuous dose of } 1 \mathrm{mg} / \text { hour } \\
\text { after standard dose of } 5-20 \mathrm{mg}\end{array}$ & $30-60 \mathrm{mg} 4^{\text {th }}$ hourly & $\begin{array}{l}50-100 \text { mg every } 4-6 \text { hours } \\
\text { Maximum dose- } 400 \mathrm{mg} \\
\text { in } 24 \text { hours }\end{array}$ \\
\hline
\end{tabular}




\section{Opioids in M axillofacial Surgery Oro-facial Pain}

The use of opioid analgesics for the management of patients with chronic pain is controversial. However, randomized and double-blind clinical trials have shown that in select groups of patients with chronic pain, the daily administration of oral opioids decreases pain levels and improves quality of life. ${ }^{[6]}$ The primary reason for the clinician's reluctance to initiate long-term opioid therapy for their patients with chronic pain is the potential risk of developing opioid tolerance, dependence, or addiction. Hence, it is essential that potential patients for this type of therapy have been carefully screened and have not had a history of drug addiction.

There are certain specific advantages of using Opioids for orofacial pain control. Opioids can be administered via many more routes than NSAIDs, including transdermal, oral, intramuscular, intravenous, transmucosal, intranasal (such as butorphanol tartrate) and via other routes. Because opioids exert an analgesic effect on peripheral sensory nerves, there is the possibility that they may be administered via topical application or local infusion and thus reduce or eliminate many of the systemic side effects associated with the systemic use of these drugs. ${ }^{[6]}$

\section{MandibularThird Molar Removal}

Removal of mandibular third molar causes swelling, trismus and moderate to severe pain.Postoperative pain control can be obtained by various non steroidal antiinflammatory agents. However, they are associated with numerous side effects and are contraindicated in a significant number of patients. ${ }^{[7,}{ }^{[8]}$ In such cases opioids like Tramadol have been found to be suitable analgesic for use in moderate pain after day-case oral surgical procedures like mandibular third molar removal. Furthermore, in a study addressing extremely severe postoperative pain, both tramadol and morphine were successful in providing pain relief. ${ }^{[7],[9]}$

In a study by Cecchetiand Peres, it has been suggested that local administration of $100 \mathrm{mg}$ tramadol contributes to provide a pain free period of 3.5-4 hours after mandibular third molar extraction, with rare adverse effects and good

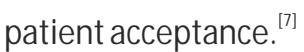

In a study conducted by M ishra et al. on 74 patients, who underwent third molar extractions, they concluded that tramadol is equally effective as ketorolac in relieving pain in the first 6 hours after molar extraction and therefore can be tried in patients who are intolerant to nonsteroidal antiinflammatory drugs. ${ }^{[10],[11]}$

In a study conducted on rats Yong-M in Liu et al. concluded that tramadol was associated with decreased IL-6 and unchanged IL-2 levels, thus suggesting that tramadol may supress the inflammation induced by incision and has beneficial role in the modulation of IL-2 associated cell mediated immunity. ${ }^{[12]}$

These studies strongly justify the use of opioids following mandibular third molar removal,providing long term analgesia along with possible anti-inflammatory effects.

\section{Orthognathic Surgery}

Orthognathic Surgery is a well established procedure that produces a significant amount of post operative pain requiring narcotic medication. Among the orthognathic surgical procedures, bimaxillary osteotomy surgeries that comprise bilateral sagittal split ramus osteotomy and Le Fort I osteotomy at the same session, have been reported to have the highest postoperative pain scores compared with other oral and maxillofacial procedures. ${ }^{[13]}$ Various studies have provided substantial evidence regarding the utility of opioids in Orthognathic patients.

In a study by Tuzuner et al, they compared the efficacy of preoperative diclofenac and tramadol with a placebo. They found that the preoperative dosage effectively decreases postoperative opioid consumption ${ }^{[13]}$ According to them, preoperative administration of $50 \mathrm{mg}$ tramadol (intravenous) and 75mg diclofenac sodium (intramuscular) in orthognathic surgery patients undergoing bimaxillary osteotomy are effective and alternative postoperative pain management methods.

A study by Cillo and Dattilo shows that a one time 
preoperative dose of pregabalin and celecoxib before adult maxillomandibular advancement surgery for obstructive sleep apnea decreased mean intravenous morphine consumption, mean daily narcotic pill consumption and mean patient perceived pain. ${ }^{14}$

In another study by Ahiskaliogluet al, forty patients were elected toundergo double jaw surgery. The study revealed that a single $150 \mathrm{mg}$ dose of pre emptivepregabalin decreased postoperative opioid consumption in the first 24 hours after double jaw surgery. ${ }^{[15]}$

It is henceforth an accepted consensus among the maxillofacial surgeons that the use of opioid analgesics is an excellent mode of pain control after orthognathic procedures.

\section{Temporo-mandibular Joint Disorders}

The long term treatment of patients with chronic temporomandibular joint dysfunction has been challenging. The long term use of opioids has been evidently found useful, leading to reduced joint pain, improved function and improved quality of life. One group of patients with chronic temporomandibular joint pain, for whom both invasive and non invasive modalities have failed, might benefit from the long term use of opioids.

A study by Kunjur et al used intra-articular morphine infusion for TMJ Arthrocentesis. They described significant

\section{References}

1. Swift JQ. Nonsteroidal Anti-Inflammatory Drugs and Opioids: Safety and Usage Concerns in the Differential Treatment of Postoperative Orofacial Pain

2. Jaffe JH, Martin WR. Opioid analgesics and antagonists, in Gilman AG, Rall TW, Nies AS, et al : Goodman and Gilman's the Pharmacological Basis of Therapeutics (ed 8). Elmsford, NY, Pergamon Press, 1990, p 485

3. Acute Pain Management Guideline Panel: Acute pain management in infants, children, and adolescents: Operative and medical procedures. Quick reference guide for clinicians. Rockville, MD, AHCPR Pub. No. 92 0020. Agency for Health Care Policy and Research, Public Health Service, US Department of Health and Human Services, 1992

4. Coulthard $\mathrm{P}$, Patel $\mathrm{N}$, Bailey N, Coulthard MB.Measuring pain after oral surgery.Oral Surgery, 2014;7;4

5. Opioids: Overview, Uses and Management of Acute and Chronic Pain

6. James SW, Roszkowski MT. The Use of Opioid Drugs in Management of Chronic Orofacial Pain

7. Ceccheti MM, Negrato GV, Peres MP, Deboni MC, NaclérioHomemMda G. Analgesic and adjuvantanestheticeffect of submucosaltramadol after mandibular third molar surgery. Oral Surg benefit of intra-articular morphine injection after arthrocentesis of the TMJ based on 405 patients. ${ }^{[16]}$

Ziegler et al designed a study to evaluate the analgesic efficacy and safety of repeated intra-articular morphine application in management of TMJ pain. They recommended that intra-articular morphine administration of $10 \mathrm{mg}$ dose provided the best pain control in cases of joint pain. ${ }^{[17]}$

Though the management of pain in Temporomandibular disorders is still debatable, the review of literature has provided considerable evidence for use of opioids for the same.

\section{Conclusion}

Management of pain can seem relentless at times and difficult to obtain. Opioids have been shown to be more efficacious in controlling pain than the myriad of other pharmacologic agents available. Resolution of pain that yields definitive improvement of quality of life and restoration of function more than justifies the overstated risks associated with chronic opioid therapy. With judicious use, opioid therapy should be considered for the management of orofacial pain that has been refractory to other methods of treatment. Administration of opioids may be a successful means of decreasing the patient's debilitating pain to tolerable levels, enabling an improvement in the quality of life and return to function.

Oral Med Oral Pathol Oral Radiol. 2014 Mar;117(3)

8. Grossi GB, Maiorana C, Garramone RA, Borgonovo A, Creminelli L, Santoro F. Assessingpostoperativediscomfortafterthird molar surgery: a prospective study. J Oral Maxillofac Surg.2007 May;65(5):901-17.

9. Wiebalck A, KulkaP ,Zenz M.Efficacy and safety of tramadol and morphine in patients with extremely severe postoperative pain. Acute Pain , September 2000; 3 (3); 22-28

10. Mishra $H$, Khan FA. A double-blind, placebo-controlled randomized comparison of pre and postoperative administration of ketorolac and tramadol for dental extraction pain. J AnaesthesiolClinPharmacol. 2012;28(2):221-225

11. Singh $P$, Rastogi $S$, Bansal $M$ et al. A Prospective Study to Assess the Levels of Interleukin-6 Following Administration of Diclofenac, Ketorolac and Tramadol After Surgical Removal of Lower Third Molars. J Maxillofac Oral Surg. 2015 Jun; 14(2): 219-225.

12. Liu Y-M, et al. Effect of tramadol on immune responses and nociceptive thresholds in a rat model of incisional pain. J Zhejiang UnivSci B. 2008;9(11):895-902.

13. Tuzuner AM, Ucok C, Kucukyavuz Z, Alkis N, Alanoglu Z.Preoperatıve DiclofenacSodium and Tramadol for Pain Relief After 
BimaxillaryOsteotomy. J Oral M axillofacSurg, December 2007; 65 (12); 2453-2458

14. Cillo Jr. JE, Dattilo DJ. Pre-Emptive Analgesia WithPregabalin and Celecoxib Decreases Postsurgical Pain Following Maxillomandibular Advancement Surgery: A Randomized Controlled Clinical Trial. J Oral M axillofacSurg, October 2014; 72(10); 1909-1914

15. Ahiskalioglu A, Ince I, Aksoy M et al.Effects of a Single-Dose of PreEmptivePregabalin on Postoperative Pain and Opioid Consumption After Double-Jaw Surgery: A Randomized Controlled Trial.
16. Kunjur J, Anand R, Brennan PA, llankovan V. An audit of 405 temporomandibular joint arthrocentesis with intra-articular morphine infusion. Oral Surg Oral Med Oral Pathol Oral RadiolEndod 1997:83:134-42

17. Ziegler CM , WiechnikJ. Analgesic Effects of Intra-Articular M orphine in Patients WithTemporomandibular Joint Disorders: A Prospective, Double-Blind, Placebo-Controlled Clinical Trial. J Oral M axillofacSurg, March 2010; 68 (3); 622-627. 